\title{
Infiltrating Bladder Urothelial Carcinoma, Plasmacytoid Variant
}

National Cancer Institute

\section{Source}

National Cancer Institute. Infiltrating Bladder Urothelial Carcinoma, Plasmacytoid Variant. NCl Thesaurus. Code C39823.

A variant of infiltrating bladder urothelial carcinoma. It is characterized by plasmacytoidlike features. 